Research

\title{
Does circulating progesterone mediate the associations of single nucleotide polymorphisms in progesterone receptor (PGR)-related genes with mammographic breast density in premenopausal women?
}

\author{
Favour A. Akinjiyan' ${ }^{1}$ Yunan Han ${ }^{2,3}$ • Jingqin Luo ${ }^{2,4}$. Adetunji T. Toriola ${ }^{2,4}$ \\ Received: 24 July 2021 / Accepted: 28 September 2021 \\ Published online: 03 November 2021 \\ (c) The Author(s) 2021 OPEN
}

\begin{abstract}
Progesterone is a proliferative hormone in the breast but the associations of genetic variations in progesterone-regulated pathways with mammographic breast density (MD) in premenopausal women and whether these associations are mediated through circulating progesterone are not clearly defined. We, therefore, investigated these associations in 364 premenopausal women with a median age of 44 years. We sequenced 179 progesterone receptor (PGR)-related single nucleotide polymorphisms (SNPs). We measured volumetric percent density (VPD) and non-dense volume (NDV) using Volpara. Linear regression models were fit on circulating progesterone or VPD/NDV separately. We performed mediation analysis to evaluate whether the effect of a SNP on VPD/NDV is mediated through circulating progesterone. All analyses were adjusted for confounders, phase of menstrual cycle and the Benjamini-Hochberg false discovery (FDR) adjusted p-value was applied to correct for multiple testing. In multivariable analyses, only PGR rs657516 had a direct effect on VPD (averaged direct effect estimate $=-0.20,95 \% \mathrm{Cl}=-0.38 \sim-0.04$, p-value $=0.02$ ) but this was not statistically significant after FDR correction and the effect was not mediated by circulating progesterone (mediation effect averaged across the two genotypes $=0.01,95 \% \mathrm{Cl}=-0.02 \sim 0.03, \mathrm{p}$-value $=0.70$ ). Five SNPs (PGR rs11571241, rs 11571239, rs1824128, rs11571150, PGRMC1 rs41294894) were associated with circulating progesterone but these were not statistically significant after FDR correction. SNPs in PGR-related genes were not associated with VPD, NDV and circulating progesterone did not mediate the associations, suggesting that the effects, if any, of these SNPs on MD are independent of circulating progesterone.
\end{abstract}

Keywords Progesterone · Genetics · Mammographic breast density · SNPs · Breast · Premenopausal

Jingqin Luo and Adetunji T. Toriola Equal contribution

Supplementary Information The online version contains supplementary material available at https://doi.org/10.1007/s12672-02100438-1.

$\triangle$ Adetunji T. Toriola, a.toriola@wustl.edu|'Medical Scientist Training Program, Washington University School of Medicine, St. Louis, MO 63110, USA. ${ }^{2}$ Division of Public Health Sciences, Department of Surgery, Washington University School of Medicine, Campus Box 8100, 660 South Euclid Ave, St. Louis, MO 63110, USA. ${ }^{3}$ Department of Breast Surgery, First Hospital of China Medical University, Shenyang 110001, Liaoning Province, China. ${ }^{4}$ Alvin J. Siteman Cancer Center at Barnes-Jewish Hospital and Washington University School of Medicine, St. Louis, MO 63110, USA.

Discover Oncology $\quad$ (2021) 12:47 $\quad$ https://doi.org/10.1007/s12672-021-00438-1 


\section{Introduction}

Mammographic breast density (MD), which refers to the amount of epithelial and stromal tissues in relation to adipose tissue in the breast [1] is a strong risk factor for breast cancer with odds ratio as high as 4-6 for women with $>75 \%$ dense tissue in their breasts compared to those with $<5 \%$ dense tissue [2, 3]. Progesterone regulates breast development [4, 5] and can cause proliferation within the breasts [6]. In preclinical models, estrogen/progesterone stimulates expansion of the mammary stem and progenitor cells, generating denser mammary morphology [7]. Studies have demonstrated that progesterone is associated with MD and breast cancer risk [8-13]. In the Women's Health Initiative trial, 12 months of estrogen/progestin use was associated with a 5\% increase in MD [14], and subsequent follow-up indicated higher breast cancer incidence in women on estrogen/progestin [15].

Progesterone exerts its functions by binding to the progesterone receptor (PGR), a nuclear receptor which, when activated, results in changes in gene expression of various proliferative genes $[16,17]$. Single nucleotide polymorphisms (SNPs) in the PGR gene modulate changes in MD in response to menopausal hormone therapy. However, there are conflicting results on the associations of genetic variations in progesterone-related pathways with MD [18-20]. In addition, there are limited data in premenopausal women [19], where hormonal variation during menstrual cycle makes it challenging to evaluate these associations. Nonetheless, it is important to investigate the associations of SNPs in the PGR gene with MD in premenopausal women as this could identify biological pathways that can be targeted in breast cancer prevention in younger women. Further, although the associations of SNPs in the PGR gene with MD could be primary i.e. directly through modulation of gene expression, or indirect, mediated by circulating progesterone levels, to the best of our knowledge, no studies have investigated these potential mediating effects. Finally, there are limited studies on how determinants of MD influence PGR SNPs. Understanding these could provide additional insight into how PGR SNPs are associated with MD. Our objectives in this study are threefold: (i) to investigate the associations of genetic variations in PGR and PGR-related SNPs (PGR Membrane Component 1 (PGRMC1) with MD in premenopausal women; (ii) determine whether these associations are mediated by circulating progesterone levels; (iii) evaluate the associations of PGR and PGR-related SNPs with known determinants of MD (such as body mass index).

\section{Materials and methods}

\subsection{Study population}

We recruited 383 healthy premenopausal women who were scheduled for an annual screening mammogram at the Joanne Knight Breast Health Center at Siteman Cancer Center at Washington University School of Medicine, (St. Louis, MO) in 2016. Our previous work provides a detailed description of the study population [21]. In brief, premenopausal women who were scheduled for their annual screening mammography at the $\mathrm{BHC}$ were mailed study flyers by research coordinators two weeks to one month in advance. Follow-up calls were then within 7 days of the scheduled appointments to screen interested individuals, provide further details and answer questions on the study [21]. Inclusion criteria include (i) premenopausal status. We identified women as premenopausal if they had a regular menstrual period within the preceding 12 months, no prior history of bilateral oophorectomy, and not used menopausal hormone therapy, (ii) No serious medical conditions that could prevent participant from returning for annual mammogram in 12 months, and (iii) not pregnant at the time of study entry. Women with previous cancer history, previous breast surgery and use of selective estrogen receptor modulators (SERM) within the last 6 months were excluded (Fig. 1).

Study participants were asked to fast on the day of their annual screening mammogram. On the day of the mammogram, blood samples were collected from each participant within a few hours of their mammogram. Height and weight measurements were collected after mammogram using a stadiometer and OMRON Full Body Sensor Body Composition Monitor and Scale model HBF-514C, respectively. Blood samples were stored at $-80^{\circ} \mathrm{C}$ within $30 \mathrm{~min}$ of collection at the Tissue Procurement Core at the Siteman Cancer Center. Participants also completed a questionnaire on determinants of MD and risk factors for breast cancer. All participants provided informed consent. The study was approved by the Institutional Review Board of the Washington University School of Medicine. 


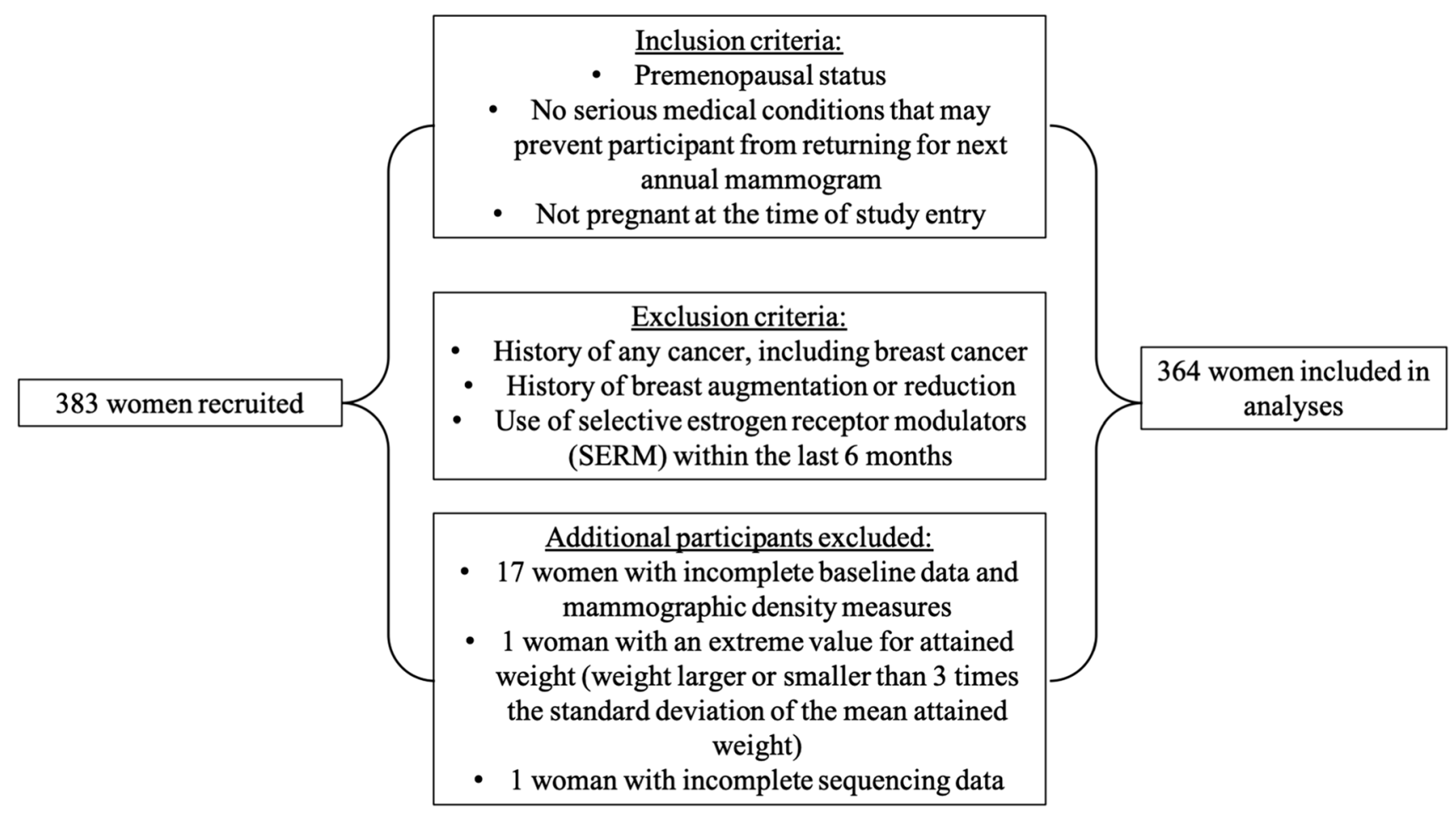

Fig. 1 Schematic depicting inclusion and exclusion criteria for study participants

\subsection{Single nucleotide polymorphisms in progesterone receptor (PGR)-related genes}

Sequencing was performed at the McDonald Genome Institute, Washington University. Automated dual indexed libraries were constructed with 100-250 ng of genomic DNA utilizing the KAPA HTP Library Kit (KAPA Biosystems) on the SciClone NGS instrument (Perkin Elmer) targeting 250 bp inserts. 365 women had genomic DNA yield of at least 100, from which libraries were pooled at an equimolar ratio yielding $\sim \mu \mathrm{g}$ per library pool prior to the hybrid capture. Library pools were hybridized with an IDT xGen Predesigned Gene Capture Pool (Integrated DNA Technologies) covering 32 genes of the human genome. The concentration of each captured library pool was accurately determined through qPCR (Kapa Biosystems) to produce cluster counts appropriate for the HiSeq4000 platform (Illumina). $2 \times 151$ bp paired end sequence data was produced according to Illumina manufacturer specification to generate approximately 1-2 Gb per IDT Targeted Capture sample, ensuring $>95 \%$ of the targets covered at a minimum of $500 \times$ depth of coverage. Variants were called using GATK, annotated by ENSEMBLE VEF and stored in variant call format. snpEff (version 4.0, build 2014-09-13) was used to further annotate the variants using human genome build 37 [22]. The R package "vcfR" was utilized to read in data to extract genotype and relevant quality measures [23]. Complete sequencing data was available for 364 women, who were included in the current analyses.

\subsection{Circulating progesterone levels}

Circulating progesterone levels were assayed at the Department of Laboratory Medicine, Boston Children's Hospital. Progesterone levels were assayed using a competitive electrochemiluminescence immunoassay on the Roche $\mathrm{E}$ Modular system (Roche Diagnostics, Indianapolis, IN). The assay is FDA-approved for clinical use and has been used in previous studies $[24,25]$. The intra-assay coefficients of variation were $2.9 \%$ at $0.73 \mathrm{ng} / \mathrm{mL}$ and $0.9 \%$ at $32.4 \mathrm{ng} /$ $\mathrm{mL}$ based on blind replicates in our samples. The inter-assay coefficients of variation were $4.8 \%$ at $0.73 \mathrm{ng} / \mathrm{mL}$ and $2.0 \%$ at $35.3 \mathrm{ng} / \mathrm{mL}$. Sensitivity was $0.15 \mathrm{ng} / \mathrm{mL}$ and specificity was 0.81 for the immunoassay. 


\subsection{Mammographic breast density assessment}

We used Volpara version 1.5 (Matakina Technology Ltd) to determine volumetric measures of MD, and we focused on volumetric percent density (VPD) and non-dense volume (NDV) in this study. VPD is the ratio of the volume of fibroglandular tissue (i.e., dense volume) to the total breast volume, expressed as a percentage. Volpara uses a computerized algorithm that calculates the $X$-ray attenuation at each pixel and converts the attenuation to an estimate of the tissue composition to create a density map $[21,26,27]$ and averages the cranial-caudal and mediolateral oblique views of the left and right breasts $[28,29]$. Corresponding to the four categories $[(\mathrm{a})-(\mathrm{d})]$ of the Breast Imaging Reporting and data System (BI-RADS; 5th edition) [30, 31], Volpara VPD measures translate to: $<3.5 \%$ (a, almost entirely fatty breasts); $3.5-7.5 \%$ (b, scattered areas of fibroglandular density); $7.5-15.5 \%$ (c, heterogeneously dense breasts); $15.5-34.5 \%$ (d, extremely dense breasts) [21, 32].

\subsection{Statistical analyses}

We sequenced 179 SNPs in PGR-related pathways. The SNPs were subject to quality control, removal of synonymous variants, filtering to keep SNPs with 10 or more samples each having at least 5 reads. Each SNP was coded using the dominant mode of inheritance into genotype $=1$ in the presence of at least one minor allele and genotype $=0$ in the presence of two of the major alleles. Genotype counts and percentages were summarized for each SNP. The associations between SNPs and between a SNP and categorical variables were investigated using Fisher's exact test. Circulating progesterone, VPD and NDV were analyzed in logarithm scale to ensure normality. The variables included in multivariable analyses were: (i) age (continuous), (ii) current BMI (continuous), (iii) family breast cancer history (Yes/ No), (iv) body shape at age 10 based on the Stunkard [33] 9-level figure pictogram categorized into 4 groups: body size 1 or 2 , body size 3 or 4 , body size 5 , and body size 6 or higher, (v) composite score of age at 1 st birth and parity (age at 1 st birth $<25$ and 3 or more kids vs. age at 1 st birth $\geq 25$ and 3 or more kids vs. age at 1 st birth $\geq 30$ and 1 or 2 kids vs. age at 1 st birth in $25 \sim 29$ and $1 \sim 2$ kids vs. nulliparous), (vi) phase of menstrual cycle (luteal vs. secretory vs. unknown).

For direct associations of SNPs with quantitative circulating progesterone and MD, linear regression models were fit on circulating progesterone or MD separately, with a SNP alone or additionally with incorporation of the six covariates. The linear coefficient estimate associated standard error (SE) and Wald test P value on the SNP were reported from the linear models. Wilcoxon test was also used to test the difference in circulating progesterone and MD by genotype, which yielded similar results to fitting linear regression. For indirect association analysis, the mediation analysis was performed to evaluate the potential mediator role of circulating progesterone, i.e., SNP effecting on MD through circulating progesterone. The mediator model in the mediation analysis fits circulating progesterone on a SNP to estimate the effect of a SNP on circulating progesterone, with inclusion of the six covariates. The outcome model in the mediation analysis, while adjusting for covariates, fits MD on circulating progesterone, a SNP under analysis and the interaction of circulating progesterone and SNP in consideration of potentially differing effect of genotypes to examine homogeneity/heterogeneity between genotypes. We report from the mediation analysis: (i) the averaged direct effect (ADE) which was estimated as the coefficient of a SNP for each genotype in the outcome model, (ii) the averaged causal mediation effect (ACME) which was estimated as the coefficient of mediator circulating progesterone in the outcome model multiplying the coefficient of a SNP in the mediator model, and (iii) the total effect which was essentially the sum of ADE and ACME, all accompanied with $95 \%$ confidence interval $(\mathrm{Cl})$ and $p$-value. For single SNP association analyses, we focused on SNPs with a genotype $=1$ count $\geq 10$ and frequency of $3 \%$ or higher.

The SKAT [34] (sequence kernel association test, with default linear weighted kernel and weight function) approach was used to investigate the overall gene set/pathway significance integrating SNPs on the PGR gene and the PGRMC1 gene, alone and collectively representing the PGR pathway, without and with inclusion of the six covariates. SNPs with high missing rate (SKAT default $=15 \%$ ) were excluded from analyses while other SNPs with lower missing rates were imputed inherently in the SKAT analyses by Hardy-Weinberg equilibrium. The Benjamini-Hochberg false discovery (FDR) adjusted $P$ value was applied to correct for multiple testing. All statistical analyses were conducted using the statistical programing language $R$ (version 3.3.1) [35] and the $R$ packages SKAT (version 1.2.1) and mediation (version 4.4.6) $[36,37]$ was employed for the SKAT analysis and mediation analysis, respectively. All tests were 2-sided unless noted otherwise. A P-value less than $5 \%$ was considered statistically significant. 


\section{Results}

\subsection{Characteristics of study participants}

Our study included a total of 364 women. Study participants were mostly non-Hispanic Whites (66.5\%) and African Americans (28.3\%) (Table 1). The median age was 48 (IQR: $44 \sim 51$ ) years. The median body mass index (BMI) was $28.1 \mathrm{~kg} / \mathrm{m}^{2}$ (IQR:24.1 34.9). The median age at first birth was 26 years (IQR: $21 \sim 30$ ) years and $26.1 \%$ of the women had a family history of breast cancer. Medium serum progesterone level was $0.3 \mathrm{ng} / \mathrm{mL}$ (IQR $0.2 \sim 3.2$ ). Median VPD was $7.3 \%$ (IQR:4.7 12.3) and BI-RADS ${ }^{\circledR}$ category $b$ (scattered areas of fibroglandular density) was the most common $(41.2 \%)$ mammographic breast density category.

\subsection{Correlations between circulating progesterone, volumetric percent density, and non-dense volume}

$\mathrm{VPD}$ and NDV were negatively correlated (Spearman correlation $=-0.78, \mathrm{P}<2.2 \mathrm{E}-16$ ). circulating progesterone was weakly positively correlated with VPD (Spearman correlation $=0.21, \mathrm{P}=5.34 \mathrm{e}-05$, Fig. 2a).

and negatively correlated with NDV (Spearman correlation $=-0.19, \mathrm{P}=0.0003$ ). When stratified by VPD categories, the lowest level of circulating progesterone (median, $\mathrm{IQR}=0.14,0.11 \sim 0.26$ ) was observed in the lowest VPD category (VPD $\leq 3.5 \%$ ) compared with each of the three higher categories (median, IQR $=0.28,0.16 \sim 1.98$, adjusted $\mathrm{P}=0.00074$ for $3.5 \% \leq \mathrm{VPD}<7.5 \%$; and $=0.49,0.16 \sim 5.66$, adjusted $\mathrm{P}=0.00011$ for $7.5 \% \leq \mathrm{VPD}<15.5 \% ;=0.34,0.20 \sim 3.89$, adjusted $\mathrm{P}=0.00011$ for $\mathrm{VPD} \geq 15.5 \%$, respectively, , Online Resource 1 ).

\subsection{Associations of SNP sets with circulating progesterone, volumetric percent density, and non-dense volume}

After initial QC filtering, 162 SNPs were available for analyses; 140 for PGR and 22 for PGRMC1 (see Online Resource 2 for SNP information and genotype count and frequency). We used the SKAT approach to test the overall associations between circulating progesterone of the SNP set on both PGR and PGRMC1 and each gene alone. In univariate analysis, PGR pathway SNPs (from both PGR and PGRMC1) were collectively associated with VPD (SKAT P=0.013) and NDV (SKAT $P=9.46 E-05)$ but not circulating progesterone (SKAT $P=0.473$ ) (Online Resource 1). The significant association with VPD was contributed by SNPs in the PGR gene (SKAT P $=0.017$ ) but not SNPs in the PGRMC1 gene (SKAT $P=0.113$ ). Both PGR SNPs and PGRMC1 SNPs were each associated with NDV (PGR: SKAT $P=0.00026 ;$ PGRMC1: SKAT $P=0.006)$ and neither with circulating progesterone (PGR: SKAT $P=0.390 ; P G R M C 1$ : SKAT $P=0.846)$. After adjusting for confounders, the SKAT analyses were not statistically significant for both PGR and PGRMC1.

\subsection{Associations of SNPs with circulating progesterone, volumetric percent density, and non-dense volume}

We investigated the associations of each SNP (with a genotype $=1$ count $>=10$ and frequency $\geq 3 \%$ ) with circulating progesterone, VPD, and NDV without (Table 2) and with (Table 3, Online Resource 3) adjustment for confounders. Fourteen SNPs, 12 on PGR and 2 on PGRMC1, were statistically significant with unadjusted $p$-values $<5 \%$ (Table 2; Fig. 3) in univariate analyses. Of these, PGR rs 1824128 was significantly associated with VPD (estimate $=0.32, \mathrm{SE}=0.13$, $\mathrm{p}$-value $=0.012$ ), NDV (estimate $=-0.35, \mathrm{SE}=0.15, \mathrm{p}$-value $=0.021$ ) and circulating progesterone (estimate $=-1.02$, $\mathrm{SE}=0.38$, p-value $=0.009$ ). In multivariable analyses, one SNP ( $r$ 657516) for VPD, none for NDV and five SNPs for circulating progesterone had p-values $<0.05$ but none was statistically significant after FDR correction with the FDRcorrected p-values of 0.08 for the 4 PGR SNPs (Table 3). Six SNPs: 4 on PGR (rs10895054, rs1 1224565, rs 11571147, and rs11571154) and 2 on PGRMC1 (rs2499040, rs2499041) were significantly associated with NDV in univariate analyses after FDR correction (Table 2c), but not after adjusting for confounders. 
Table 1 Characteristics of study participants $(n=364)$ recruited at the Joanne Knight Breast Health Center at Siteman Cancer Centre at Washington University School of Medicine, (St. Louis, MO) in 2016

\begin{tabular}{l} 
Characteristic \\
\hline Age (years) \\
Progesterone (ng/mL) \\
Mammographic breast density \\
Volumetric percent density $(\%)^{\mathrm{a}}$ \\
$<3.5 \%$ \\
$3.5-7.5 \%$ \\
$\geq 7.5-15.5 \%$ \\
$\geq 15.5 \%$ \\
Non-dense volume $\left(\mathrm{cm}^{3}\right)$ \\
Race \\
Non-Hispanic White \\
Black or African American \\
Others \\
Missing
\end{tabular}

Age at first birth (years)

Age at second birth (years)

Parity and Age at first birth

295

228

364

Nulliparous

$1-2$ kids; $<25$

1-2 kids; $25 \sim 29$

$1-2$ kids; $>=30$

$>=3$ kids; $<25$

$>=3$ kids; $>=25$

Missing

BMI $\left(\mathbf{k g} / \mathbf{m}^{2}\right)$

364

Normal

Overweight

$>=30$

Missing

Body Shape at Age 10

$1 \sim 2$

$3 \sim 4$

5

$>=6$

Missing

Family history of breast cancer

Yes

364

364

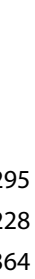

464

364

364

$\begin{array}{ll} & 34(9.3) \\ & 150(41.2) \\ & 116(31.9) \\ & 64(17.6)\end{array}$

4

$\begin{array}{ll} & 242(66.5) \\ & 103(28.3) \\ & 14(3.9) \\ & 5(1.4) \\ & 26(21 \sim 30) \\ & 29(25 \sim 33) \\ 295 & \\ 364 & 66(18.1) \\ & 63(17.3) \\ & 46(12.6) \\ & 78(21.4) \\ & 60(16.5) \\ & 48(13.2) \\ & 3(0.9) \\ & 28.13(24.1 \sim 34.9) \\ & 113(31.0) \\ 96(26.4) \\ 148(40.7) \\ 7(1.9)\end{array}$

364

$136(37.4)$

$108(29.7)$

45 (12.4)

$26(7.1)$

49 (13.5)

364

95 (26.1)

257 (70.6)

$12(3.3)$

Missing

Phase of menstrual cycle

Follicular

78 (21.4)

$123(33.8)$

$104(28.6)$

Oligomenorrhea

Missing

59 (16.2)

a Volumetric Percent Density. Volpara volumetric percent density ranges from 0.5 to $34.5 \%$. Corresponding to the four categories (a) (d) of the breast imaging reporting and data system (BI-RADS ${ }^{\oplus}$ ) (5th edition), Volpara volumetric percent density measures translate to: $<3.5$ (a-almost entirely fatty breasts); $\geq 3.5$ and $<7.5$ ( $b$ - scattered areas of fibroglandular density) $\geq 7.5$ and $<15.5$ ( $c$ - heterogeneously dense breasts); $\geq 15.5 \%$ (d-extremely dense breasts)

* Summary include median and interquartile range (IQR) for quantitative variables and count (\%) for categorical variables 

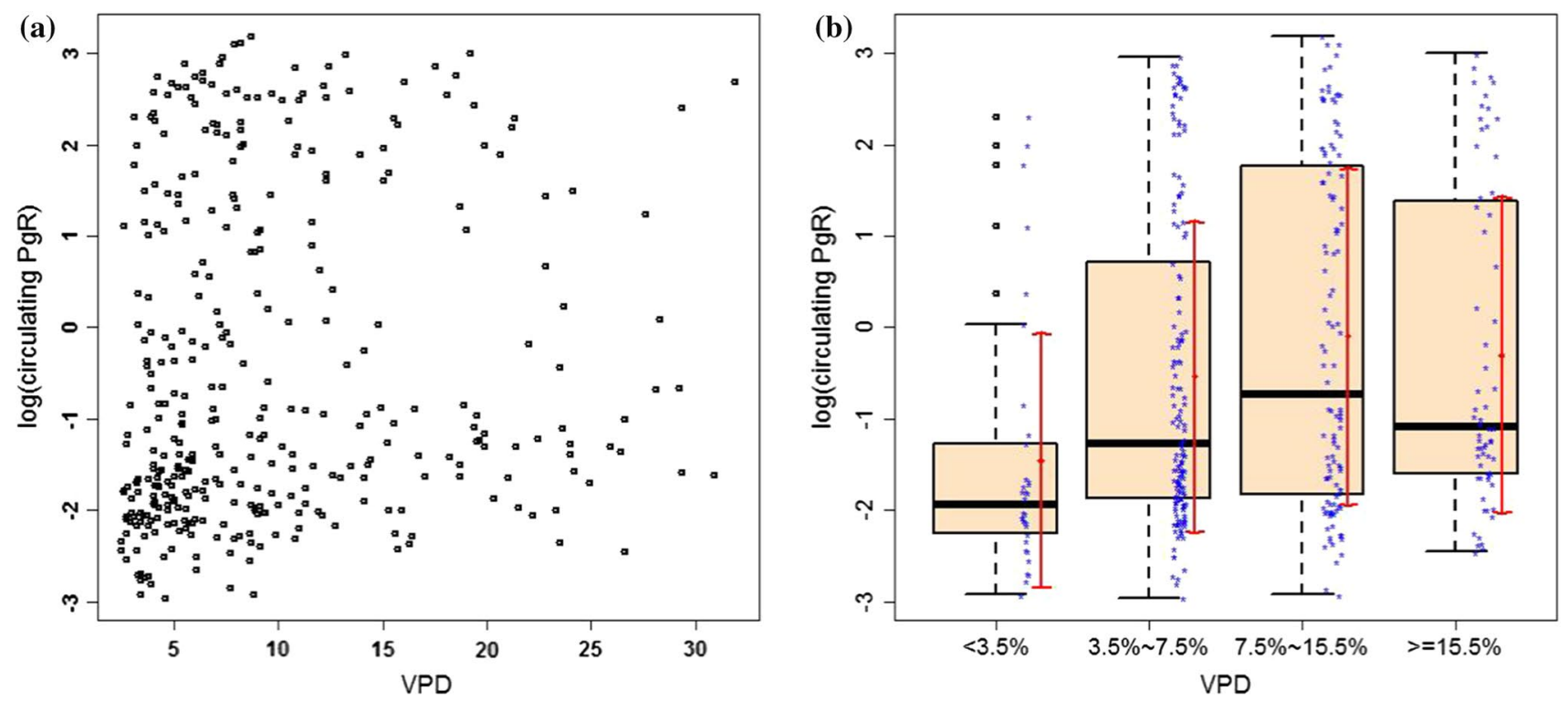

Fig. 2 a Scatterplot of $c P g R$ (in log scale) with VPD (Spearman cor $=0.21, p=5.35 E-05$ ) b Boxplot of PgR (in log scale) by VPD category (blue points represent each data point, red dot and arrow indicate mean and SD, black horizontal line indicates median). The overall Kruskal-Wallis test comparing circulating PgR by the 4 VPD categories indicated significant difference among the 4 categories $(P=9.58 \mathrm{E}-05)$ and posthoc pairwise Wilcoxon rank sum test with Benjamini-Hochberg adjustment on $\mathrm{p}$ values indicated significant difference between the lowest " $<3.5 \%$ " category with each of the three higher categories in sequential order (adjusted $\mathrm{P}=0.00074,0.00011$, and 0.00011 , respectively) but not others

\subsection{Mediation analyses of PGR SNPs, circulating progesterone with volumetric percent density, and non-dense volume}

To investigate whether circulating progesterone mediates the associations of PGR SNPs with VPD and NDV, we performed mediation analyses focusing on the 42 SNPs with genotype $=1$ count $>=10$ and frequency $>3 \%$. After adjustment for confounders, only PGR rs657516 had a direct effect on VPD (averaged direct effect estimate $=-0.20$, $95 \% \mathrm{Cl}=-0.38 \sim-0.04, \mathrm{P}=0.02$, Fig. 4) with additional adjustment for circulating progesterone and interaction between SNP and circulating progesterone besides the six covariates and the effect was homogeneous between the two genotypes, but there was no mediation effect (mediation effect averaged across the two genotypes $=0.01$, $95 \% \mathrm{Cl}=-0.02 \sim 0.03, \mathrm{P}=0.704)$.

\subsection{Associations of SNPs with determinants of mammographic breast density}

Many SNPs were associated with the determinants of MD in multivariable analyses but most were not statistically significant after FDR correction (Online Resource 4, Online Resource 5). Four SNPs: 3 on PGR (rs11224565, rs10895054, and rs11571147) and 1 on PGRMC1 (rs2499040) were associated with BMI after FDR corrections (Online Resource 4). The rs 11571153 SNP was positively associated with body shape at age 10 (30.6\% of women with at least 1 copy of the effect allele $(T)$ had a larger shape with a body shape $=5$ or $>=6$ versus $16.6 \%$ in the women without the effect allele, raw $\mathrm{P}=0.016$ ) (AOnline Resource 5). 
Table 2 Single nucleotide polymorphisms (SNPs) with significant univariate associations (without adjustment for covariates) among 42 SNPs: with genotype 1 frequency $>=3 \%$ and count $>=10$ ) with (a) circulating progesterone (cPgR), (b) volumetric percent density (VPD) and (c) non-dense volume (NDV) from linear regression

\begin{tabular}{|c|c|c|c|c|c|c|}
\hline \multicolumn{3}{|l|}{ SNPID } & \multicolumn{4}{|l|}{ cPgR } \\
\hline Gene & SNP & Minor Allele & Estimate & SE & $P$ value & FDR P-value \\
\hline PGR11 & rs1824128 & $\mathrm{T}$ & -1.02 & 0.38 & 0.009 & 0.209 \\
\hline PGR11 & rs11571150 & A & -1.16 & 0.45 & 0.010 & 0.209 \\
\hline \multicolumn{7}{|l|}{ (b) } \\
\hline \multicolumn{3}{|l|}{ SNP ID } & \multicolumn{4}{|l|}{ VPD } \\
\hline Gene & SNP & Minor Allele & Estimate & SE & $P$ value & FDR P-value \\
\hline PGR11 & rs1824128 & $\mathrm{T}$ & 0.32 & 0.13 & 0.012 & 0.149 \\
\hline PGR11 & rs10895054 & $\mathrm{T}$ & 0.26 & 0.10 & 0.012 & 0.149 \\
\hline PGRMC1 & rs2499040 & G & -0.25 & 0.10 & 0.015 & 0.149 \\
\hline PGR11 & rs2020875 & A & 0.44 & 0.18 & 0.016 & 0.149 \\
\hline PGR11 & NA & A & 0.43 & 0.18 & 0.020 & 0.149 \\
\hline PGR11 & rs11224565 & $\mathrm{T}$ & -0.29 & 0.13 & 0.021 & 0.149 \\
\hline PGR11 & rs11571147 & G & -0.30 & 0.13 & 0.027 & 0.159 \\
\hline PGRMC1 & rs2499041 & G & -0.22 & 0.11 & 0.043 & 0.216 \\
\hline PGR11 & rs1042838 & A & 0.15 & 0.08 & 0.046 & 0.216 \\
\hline \multicolumn{7}{|l|}{ (c) } \\
\hline \multicolumn{3}{|l|}{ SNP ID } & \multicolumn{4}{|l|}{ NDV } \\
\hline Gene & SNP & Minor Allele & Estimate & SE & $P$ value & FDR P-value \\
\hline PGR11 & rs1824128 & $\mathrm{T}$ & -0.35 & 0.15 & 0.021 & 0.103 \\
\hline PGR11 & rs10895054 & $\mathrm{T}$ & -0.37 & 0.12 & 0.003 & 0.028 \\
\hline PGRMC1 & rs2499040 & G & 0.39 & 0.12 & 0.002 & 0.021 \\
\hline PGR11 & rs2020875 & A & -0.42 & 0.22 & 0.052 & 0.163 \\
\hline PGR11 & NA & A & -0.49 & 0.22 & 0.025 & 0.106 \\
\hline PGR11 & rs11224565 & $\mathrm{T}$ & 0.64 & 0.14 & 0.000 & 0.000 \\
\hline PGR11 & rs11571147 & G & 0.51 & 0.16 & 0.001 & 0.021 \\
\hline PGRMC1 & rs2499041 & G & 0.35 & 0.12 & 0.005 & 0.037 \\
\hline PGR11 & rs1042838 & A & -0.15 & 0.09 & 0.110 & 0.256 \\
\hline PGR11 & rs11571255 & A & 0.38 & 0.19 & 0.043 & 0.149 \\
\hline PGR11 & rs499699 & $C$ & 0.18 & 0.08 & 0.022 & 0.103 \\
\hline PGR11 & rs11571150 & A & 0.48 & 0.19 & 0.011 & 0.065 \\
\hline PGR11 & rs11571154 & $\mathrm{T}$ & 0.51 & 0.18 & 0.005 & 0.037 \\
\hline PGR11 & rs11571153 & $\mathrm{T}$ & -0.17 & 0.08 & 0.029 & 0.109 \\
\hline
\end{tabular}

*Estimate: linear regression coefficient estimate; SE: standard error, $\mathrm{P}$ value: Wald test P; FDR P FDR adjusted P value across all SNPs.

\section{Discussion}

SNPs in the PGR and PGRMC1 genes were not associated with circulating progesterone, VPD and NDV after FDR correction. Circulating progesterone did not mediate the associations of SNPs in PGR-related genes with MD, suggesting that the effects, if any, of these SNPs are independent of circulating progesterone. Our findings should, however, be interpreted in the context of the small sample size.

To the best of our knowledge, this is one of the first studies to investigate the associations of SNPs in PGR and PGRrelated genes with MD in premenopausal women and determine whether circulating progesterone levels mediate 
Table 3 Single nucleotide polymorphisms (SNPs) with significant multivariate adjusted (for the six covariates) associations among 42 SNPs: with genotype 1 frequency $>=3 \%$ and count $>=10$ ) with $(A)$ circulating Progesterone (cPgR), (B) volumetric Percent Density (VPD)

\begin{tabular}{|c|c|c|c|c|c|c|}
\hline \multicolumn{3}{|l|}{ SNP ID } & \multicolumn{4}{|l|}{ CPgR } \\
\hline Gene & SNP & Minor Allele & Estimate & SE & $P$ value & FDR P-value \\
\hline PGR11 & rs11571241 & $\mathrm{T}$ & -1.81 & 0.63 & 0.004 & 0.076 \\
\hline PGR11 & rs11571239 & $\mathrm{T}$ & -1.81 & 0.63 & 0.004 & 0.076 \\
\hline PGR11 & rs1824128 & $\mathrm{T}$ & -1.21 & 0.44 & 0.007 & 0.076 \\
\hline PGR11 & rs11571150 & A & -1.55 & 0.57 & 0.007 & 0.076 \\
\hline PGRMC1 & rs41294894 & G & -1.81 & 0.85 & 0.037 & 0.308 \\
\hline
\end{tabular}

(b)

\begin{tabular}{|c|c|c|c|c|c|c|}
\hline \multicolumn{3}{|l|}{ SNP ID } & \multicolumn{4}{|l|}{ VPD } \\
\hline Gene & SNP & Minor allele & estimate & SE & $P$ value & FDR P-value \\
\hline PGR11 & rs657516 & G & -0.1966 & 0.0952 & 0.0399 & 0.9565 \\
\hline
\end{tabular}

*Estimate: linear regression coefficient estimate

$S E$ standard error, $P$ value Wald test $P, F D R P$ FDR adjusted $P$ value

these associations. Although there were no statistically significant associations after correcting for multiple testing, some SNPs are still worth discussing because of the associations observed prior to correcting for multiple testing. PGR rs657516 SNP had an inverse average direct effect on VPD. This SNP is located within the enhancer region (GH11J100921) on chromosome 11[38]. PGR is one of the genes regulated by this enhancer region [38]. The location of this variant at an enhancer region suggests that this SNP may affect gene expression, activity, or function of PGR. A substitution in the enhancer region for PGR may cause downregulation of PGR expression, reduce progesterone's proliferative action in the breasts and explain the observed inverse direct effect on volumetric percent density. PGR rs 1824128 was the only SNP in our study which was significantly associated with circulating progesterone, VPD and NDV. This SNP was positively associated with VPD and negatively associated with NDV and circulating progesterone and had previously been shown to be associated with an increased risk of breast cancer [39].

PGR SNPs rs 2499041 and rs 11571154 were significantly associated with NDV prior to FDR correction. Rs 2499041 is a rare intronic variant with no known clinical significance [40, 41]. PGR SNP rs 11571154 is also in the non-coding region $[40,42]$. PGR rs 11571150 , which was associated with circulating progesterone is a missense variant with high frequency (MAF 0.12) in African Americans [40,43]. PGRMC1 rs41294894 is an intron variant with increased frequency in persons of African descent $(0.13)[40,44]$. This SNP was inversely associated with circulating progesterone. The inverse association of rs 41294894 with circulating progesterone suggests that it could be associated with a reduction in breast cancer risk since serum progesterone has been associated with increased breast cancer risk [45] and deserves further evaluation in larger studies (Online Resource 6).

PGR SNPs rs 11224565, rs10895054, rs11571147 and PGRMC1 rs2499040 were associated with BMI after FDR correction. Notably, all four SNPs were also associated with VPD and/or NDV in univariate analyses. These findings suggest a possible interaction between BMI and MD mediated through PGR-related gene variants. Our sample is, however, not large enough to evaluate these hypotheses, hence, larger studies are needed to characterize these. Rs 11224565 is a noncoding variant located in the $3^{\prime} \mathrm{UTR}$ and could potentially influence BMI via alterations in gene expression [40, 46]. Prior work has shown that rs 10895054 is associated with increased risk of breast cancer in African American women [47]. BMI at age 10 is inversely associated with breast cancer risk in premenopausal women [48]. Thus, the rs10895054 SNP may provide an insight into the associations of BMI with breast cancer risk in premenopausal women. The rs 11571153 SNP [49] was positively associated with body shape at age 10, but inversely associated with NDV. This variant could play a role in early life body shape and explain in part the associations of adiposity at age 10 with MD in premenopausal women that we have previously reported [21].

Both salivary and circulating luteal phase progesterone levels are positively associated with MD in premenopausal women $[50,51]$. Other studies have shown positive associations between endogenous progesterone levels and MD [52-56]. Prior work has also shown that women with PGR + 331 GG genotype were more susceptible to the effects of 
(a)
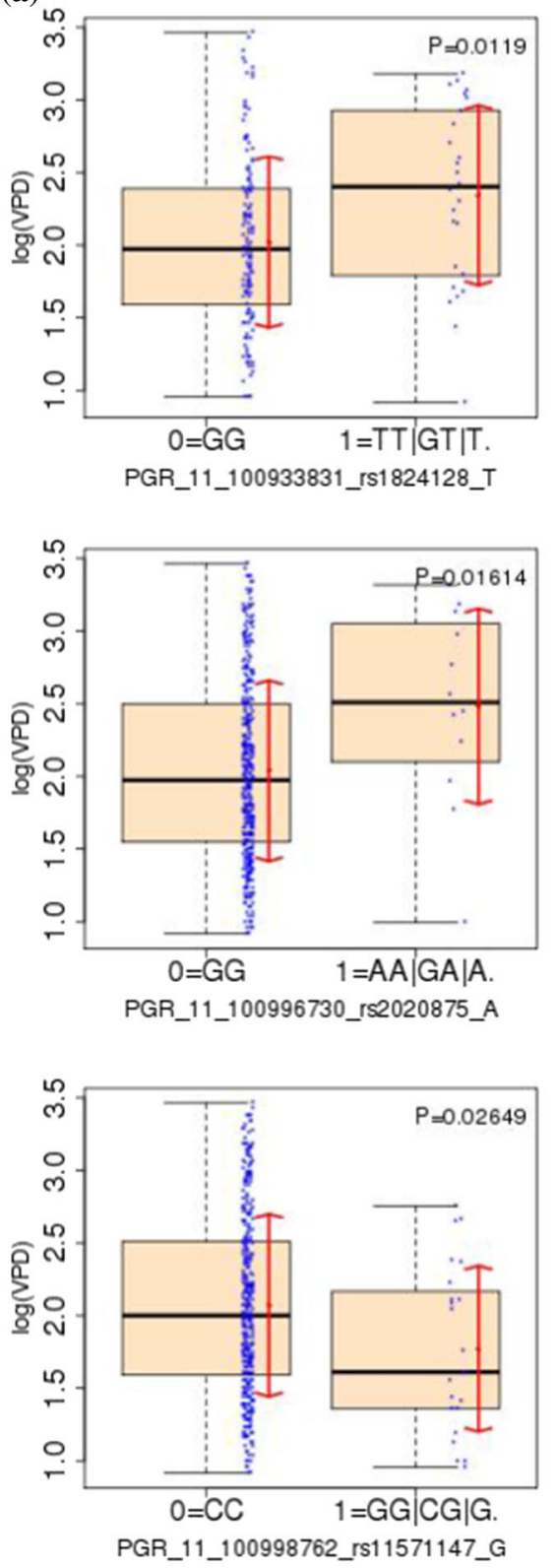

(c)

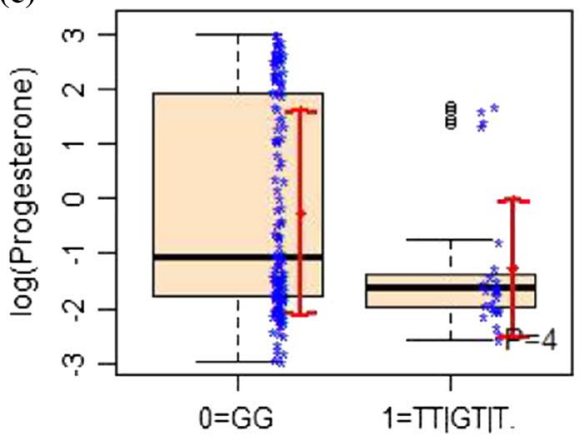

PGR_11_100933831_rs1824128_T
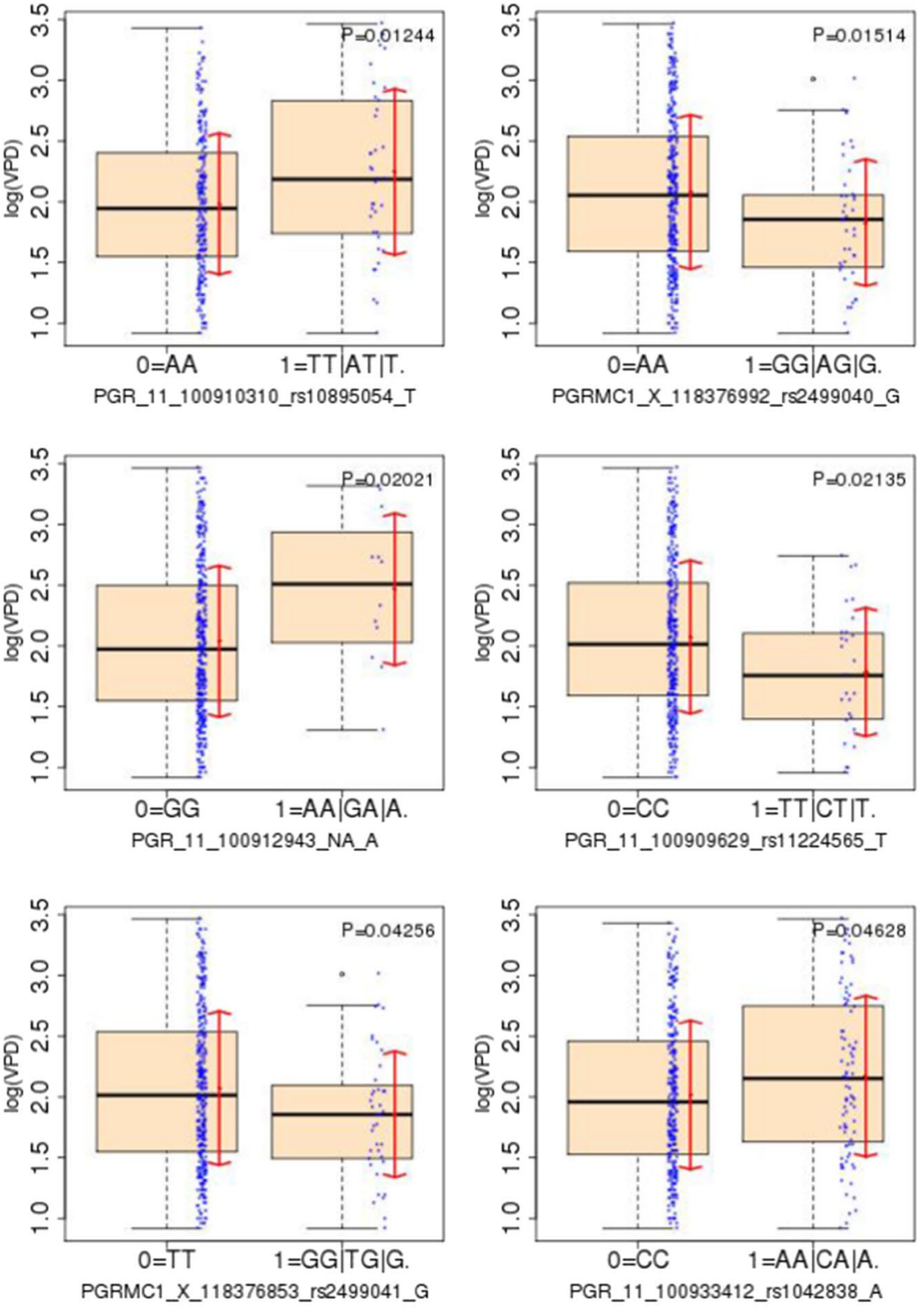

Fig. 3 Single nucleotide polymorphisms (SNPs) associated with A volumetric percent density (VPD), B non-dense volume (NDV) and C circulating progesterone (cPgR) (all in log scale) from univariate linear regression 

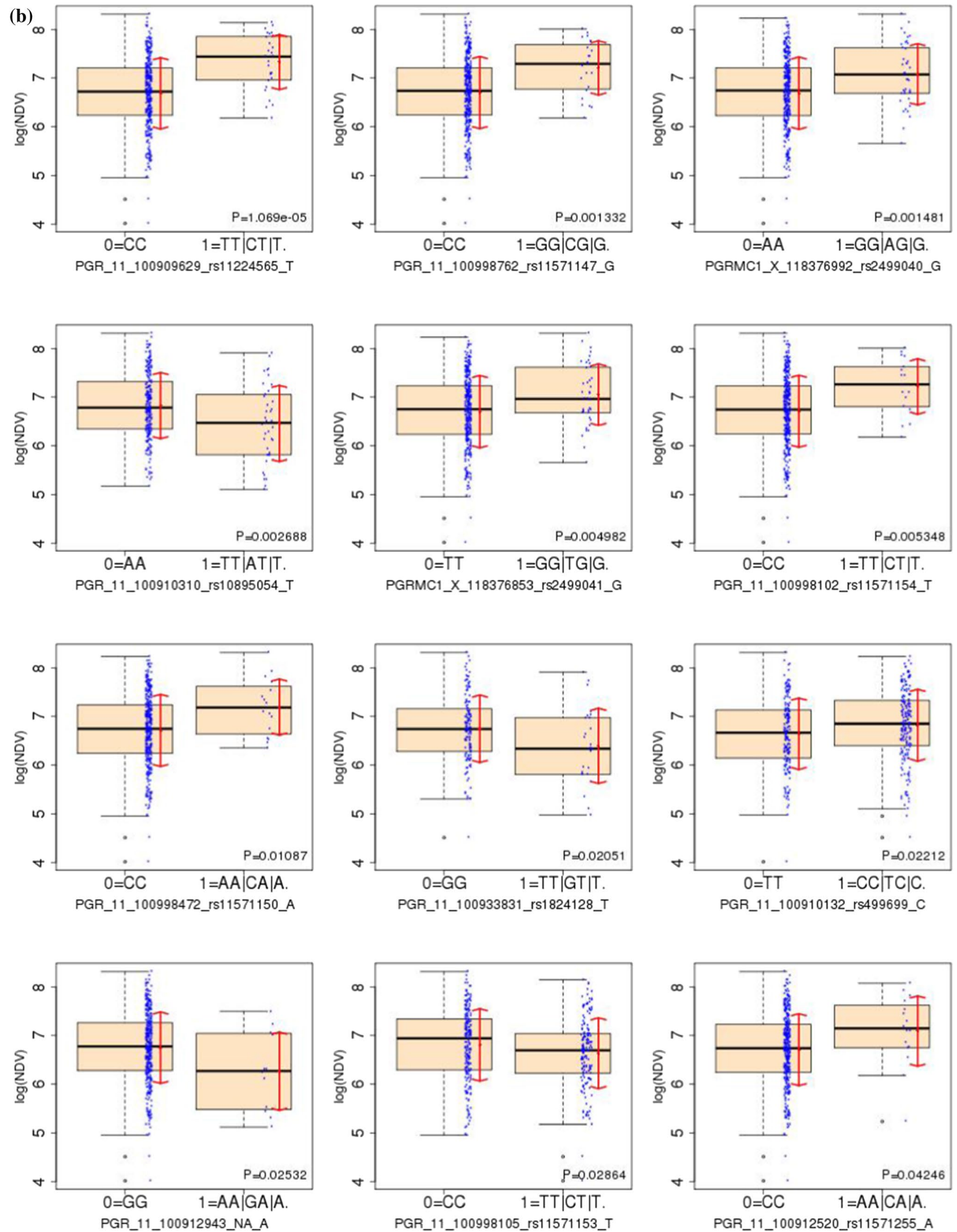

Fig. 3 (continued) 
Fig. 4 Direct effect (ADE), mediation effect (ACME) and total effect by genotype (solid line: genotype 1 of at least 1 minor allele "G"; dotted line: genotype 0 of two major alleles) of PGR_11_100922112 rs657516_G for VPD

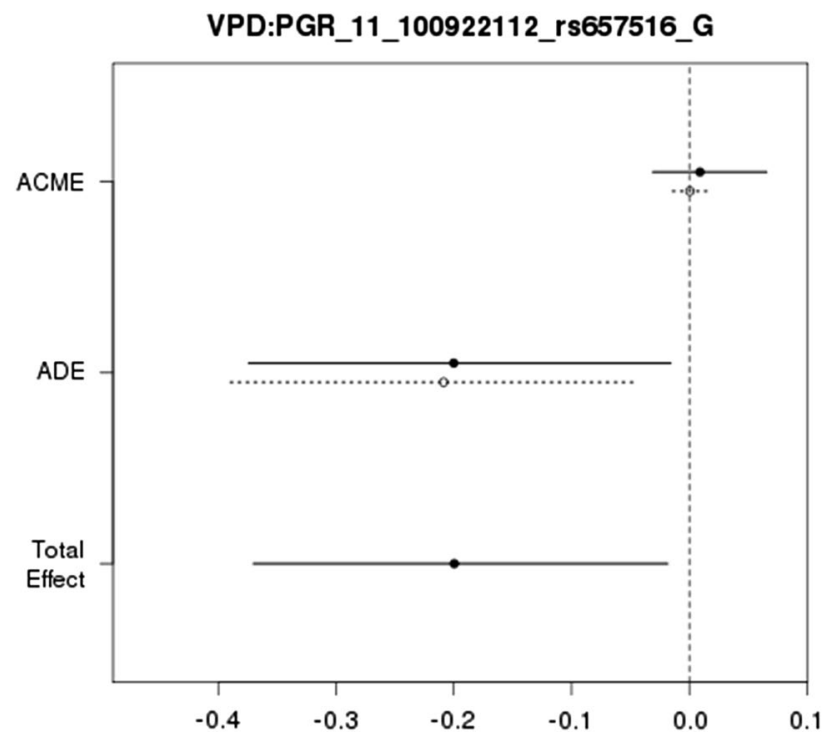

hormone therapy use on MD [19], however this study included both pre-, peri- and post-menopausal women. Our study focused on the associations between SNPs in PGR-related genes and MD in premenopausal women as well as the possible role that circulating progesterone plays in this association.

Our study has the following limitations which should be considered when interpreting the results: (i) the cross-sectional study design, (ii) our sample size was small and could explain why some results were null after FDR correction. The small sample size also precluded us from performing analyses stratified by race. In spite of these limitations, our study has the following strengths: (i) study participants were recruited among women attending annual routine screening mammogram, which enhances generalizability of our findings, (ii) we used Volpara to measure MD. Volpara provides automated, highly reproducible and robust volumetric measures of MD, (iii) we adjusted for the phase of the menstrual cycle in our analyses to account for the cyclical variation in circulating progesterone levels as circulating progesterone varies greatly by phase of menstrual cycles in premenopausal women.

In conclusion, SNPs in PGR-related genes (PGR and PGRMC1) were not associated with MD in this study population of 364 premenopausal women. Larger studies are needed to further investigate the intricate associations between progesterone signaling and MD in premenopausal women, and to confirm the associations of PGR-related SNPs with circulating progesterone and BMI.

Authors' contributions ATT conceived the project. FAA, YH, JL and ATT contributed to data analysis and interpretation. FAA wrote the main manuscript and all authors reviewed the manuscript.

Funding The study is supported by funds from the Susan G. Komen Foundation (CCR15332379-ATT), NIH/NCI (R01CA246592 and R37CA235602-ATT).

Data availability The raw datasets generated during the current study are available from the corresponding author on reasonable request. All data analysed during this study are included in this published article [and its supplementary information files].

\section{Declarations}

Competing interests The authors declare no competing interests.

Open Access This article is licensed under a Creative Commons Attribution 4.0 International License, which permits use, sharing, adaptation, distribution and reproduction in any medium or format, as long as you give appropriate credit to the original author(s) and the source, provide a link to the Creative Commons licence, and indicate if changes were made. The images or other third party material in this article are included in the article's Creative Commons licence, unless indicated otherwise in a credit line to the material. If material is not included in the article's Creative Commons licence and your intended use is not permitted by statutory regulation or exceeds the permitted use, you will need to obtain permission directly from the copyright holder. To view a copy of this licence, visit http://creativecommons.org/licenses/by/4.0/. 


\section{References}

1. Wolfe JN. Breast patterns as an index of risk for developing breast cancer. AJR Am J Roentgenol. 1976;126:1130-7.

2. McCormack VA, dos Santos SI. Breast density and parenchymal patterns as markers of breast cancer risk: a meta-analysis. Cancer Epidemiol Biomark Prev. 2006;15:1159-69.

3. Pettersson A, Graff RE, Ursin G, dos Santos Silva I, McCormack V, Baglietto L, et al. Mammographic density phenotypes and risk of breast cancer: a meta-analysis. J Natl Cancer Inst. 2014;106:dju078.

4. Lange CA, Yee D. Progesterone and breast cancer. Womens Health (Lond Engl). 2008;4:151-62.

5. Brisken C, Park S, Vass T, Lydon JP, O'Malley BW, Weinberg RA. A paracrine role for the epithelial progesterone receptor in mammary gland development. Proc Natl Acad Sci USA. 1998;95:5076-81.

6. Azeez JM, Sithul H, Hariharan I, Sreekumar S, Prabhakar J, Sreeja S, et al. Progesterone regulates the proliferation of breast cancer cells - in vitro evidence. Drug Des Devel Ther. 2015;9:5987-99.

7. Haslam SZ. Experimental mouse model of hormonal therapy effects on the postmenopausal mammary gland. Breast Dis. 2006;24:71-8.

8. Boyd NF, Dite GS, Stone J, Gunasekara A, English DR, McCredie MR, et al. Heritability of mammographic density, a risk factor for breast cancer. N Engl J Med. 2002;347:886-94.

9. Sung H, Ren J, Li J, Pfeiffer RM, Wang Y, Guida JL, et al. Breast cancer risk factors and mammographic density among high-risk women in urban China. npj Breast Cancer. 2018;4:3.

10. Topal NB, Ayhan S, Topal U, Bilgin T. Effects of hormone replacement therapy regimens on mammographic breast density: the role of progestins. J Obstet Gynaecol Res. 2006;32:305-8.

11. Gapstur SM, López P, Colangelo LA, Wolfman J, Van Horn L, Hendrick RE. Associations of breast cancer risk factors with breast density in hispanic women. Cancer Epidemiol Biomark Prev. 2003;12:1074-80.

12. McTiernan A, Martin CF, Peck JD, Aragaki AK, Chlebowski RT, Pisano ED, et al. Estrogen-plus-progestin use and mammographic density in postmenopausal women: Women's Health Initiative randomized trial. J Natl Cancer Inst. 2005;97:1366-76.

13. Azam S, Jacobsen KK, Aro AR, Lynge E, Andersen ZJ. Hormone replacement therapy and mammographic density: a systematic literature review. Breast Cancer Res Treat. 2020;182:555-79.

14. Chlebowski RT, Rohan TE, Manson JE, Aragaki AK, Kaunitz A, Stefanick ML, et al. Breast cancer after use of estrogen plus progestin and estrogen alone: analyses of data from 2 women's health initiative randomized clinical trials. JAMA Oncol. 2015;1:296-305.

15. Chlebowski RT, Anderson GL, Aragaki AK, Manson JE, Stefanick ML, Pan K, et al. Association of menopausal hormone therapy with breast cancer incidence and mortality during long-term follow-up of the women's health initiative randomized clinical trials. JAMA. 2020;324:369-80.

16. Hilton HN, Graham JD, Clarke CL. Minireview: Progesterone regulation of proliferation in the normal human breast and in breast cancer: a tale of two scenarios? Mol Endocrinol. 2015;29:1230-42.

17. Graham JD, Clarke CL. Physiological action of progesterone in target tissues*. Endocr Rev. 1997;18:502-19.

18. Chambo D, Kemp C, Costa AM, Souza NC, GuerreirodaSilva ID. Polymorphism in CYP17, GSTM1 and the progesterone receptor genes and its relationship with mammographic density. Braz J Med Biol Res. 2009;42:323-9.

19. van Duijnhoven FJ, Peeters PH, Warren RM, Bingham SA, Uitterlinden AG, van Noord PA, et al. Influence of estrogen receptor alpha and progesterone receptor polymorphisms on the effects of hormone therapy on mammographic density. Cancer Epidemiol Biomarkers Prev. 2006;15:462-7.

20. Giacomazzi J, Aguiar E, Palmero El, Schmidt AV, Skonieski G, Filho DD, et al. Prevalence of ERa-397 Pvull C/T, ERa-351 Xbal A/G and PGR PROGINS polymorphisms in Brazilian breast cancer-unaffected women. Braz J Med Biol Res. 2012;45:891-7.

21. Alimujiang A, Imm KR, Appleton CM, Colditz GA, Berkey CS, Toriola AT. Adiposity at age 10 and mammographic density among premenopausal women. Cancer Prev Res. 2018;11:287-94.

22. Cingolani P, Platts A, le Wang L, Coon M, Nguyen T, Wang L, et al. A program for annotating and predicting the effects of single nucleotide polymorphisms, SnpEff: SNPs in the genome of Drosophila melanogaster strain w1118; iso-2; iso-3. Fly (Austin). 2012;6:80-92.

23 Knaus BJ, Grünwald NJ. VCFR: a package to manipulate and visualize variant call format data in R. Mol Ecol Resour. 2017;17(1):44-53. https://doi.org/10.1111/1755-0998.12549.

24. Martínez-Ruiz A, Sarabia-Meseguer MD, Pérez-Fornieles J, Vílchez JA, Tovar-Zapata I, Noguera-Velasco JA. Placental growth factor, soluble fms-like tyrosine kinase 1 and progesterone as diagnostic biomarkers for ectopic pregnancy and missed abortion. Clin Biochem. 2014;47:844-7.

25. Wang B, Mi M, Wang J, Wei N, Zhang Q, Zhu J, et al. Does the increase of endogenous steroid hormone levels also affect breast cancer risk in Chinese women? A case-control study in Chongqing, China. Int J Cancer. 2009;124:1892-9.

26. Lee HN, Sohn YM, Han KH. Comparison of mammographic density estimation by Volpara software with radiologists' visual assessment: analysis of clinical-radiologic factors affecting discrepancy between them. Acta Radiol. 2015;56:1061-8.

27. Ellison-Loschmann L, McKenzie F, Highnam R, Cave A, Walker J, Jeffreys M. Age and ethnic differences in volumetric breast density in new zealand women: a cross-sectional study. PLoS ONE. 2013;8:e70217.

28. Voltara Solutions. Introducing Volparadensity. Wellington, New Zealand: Volpara Solutions from Matakina Technology; 2017.

29. Solutions V. Volpara density user manual version 1.5. Wellington, New Zealand: Volpara Solutions from Matakina Technology 2013

30 Rao AA, Feneis J, Lalonde C, Ojeda-Fournier H. A pictorial review of changes in the BI-RADS fifth edition. Radiographics. 2016;36:623-39.

31 D'Orsi CJ, Sickles EA, Mendelson EB, Morris EA, et al. ACR BI-RADS ${ }^{\circ}$ Atlas, breast imaging reporting and data system. Reston, VA: American College of Radiology; 2013.

32. Brandt KR, Scott CG, Ma L, Mahmoudzadeh AP, Jensen MR, Whaley DH, et al. Comparison of clinical and automated breast density measurements: implications for risk prediction and supplemental screening. Radiology. 2016;279:710-9.

33. Stunkard AJ, Sorensen T, Schulsinger F. Use of the Danish Adoption Register for the study of obesity and thinness. Res Publ Assoc Res Nerv Ment Dis. 1983;60:115-20. 
34. Wu MC, Lee S, Cai T, Li Y, Boehnke M, Lin X. Rare-variant association testing for sequencing data with the sequence kernel association test. Am J Hum Genet. 2011;89:82-93.

35. R Core Team. R Foundation for Statistical Computing, Vienna, Australia. 2017.

36. Tingley D, Yamamoto T, Hirose K, Keele L, Imai K. Mediation: R package for causal mediation analysis. J Stat Softw 2014;59(5):1-38. URL http://www.jstatsoft.org/v59/i05/

37. Imai K, Keele L, Yamamoto T. Identification, inference and sensitivity analysis for causal mediation effects. Stat Sci. 2010;25(1):51-71.

38. Rosen, N., V. Chalifa-Caspi, O. Shmueli, A. Adato, M. Lapidot, J. Stampnitzky, M. Safran, and D. Lancet. GeneLoc: Exon-based integration of human genome maps. Bioinformatics 2003;19(S1). http://genecards.weizmann.ac.il/geneloc

39. Nyante SJ, Gammon MD, Kaufman JS, Bensen JT, Lin DY, Barnholtz-Sloan JS, et al. Genetic variation in estrogen and progesterone pathway genes and breast cancer risk: an exploration of tumor subtype-specific effects. Cancer Causes Control. 2015;26:121-31.

40. Sherry ST, Ward MH, Kholodov M, Baker J, Phan L, Smigielski EM, Sirotkin K. dbSNP: the NCBI database of genetic variation. Nucleic Acids Res. 2001;29(1):308-11.

41. Database of Single Nucleotide Polymorphisms (dbSNP). Bethesda (MD): National Center for Biotechnology Information, National Library of Medicine. dbSNP accession:\{rs2499041\}, (dbSNP Build ID: \{152\}). http://www.ncbi.nlm.nih.gov/SNP/

42. Database of Single Nucleotide Polymorphisms (dbSNP). Bethesda (MD): National Center for Biotechnology Information, National Library of Medicine. dbSNP accession:\{rs11571154\}, (dbSNP Build ID: \{152\}). http://www.ncbi.nlm.nih.gov/SNP/

43. Database of Single Nucleotide Polymorphisms (dbSNP). Bethesda (MD): National Center for Biotechnology Information, National Library of Medicine. dbSNP accession:\{rs11571150\}, (dbSNP Build ID: \{152\}). http://www.ncbi.nlm.nih.gov/SNP/

44. Database of Single Nucleotide Polymorphisms (dbSNP). Bethesda (MD): National Center for Biotechnology Information, National Library of Medicine. dbSNP accession:\{rs41294894\}, (dbSNP Build ID: \{152\}). http://www.ncbi.nlm.nih.gov/SNP/

45. Sprague BL, Trentham-Dietz A, Gangnon RE, Buist DS, Burnside ES, Bowles EJ, et al. Circulating sex hormones and mammographic breast density among postmenopausal women. Horm Cancer. 2011;2:62-72.

46. Database of Single Nucleotide Polymorphisms (dbSNP). Bethesda (MD): National Center for Biotechnology Information, National Library of Medicine. dbSNP accession:\{rs11224565\}, (dbSNP Build ID: $\{152\})$. http://www.ncbi.nlm.nih.gov/SNP/

47. Gabriel CA, Mitra N, Demichele A, Rebbeck T. Association of progesterone receptor gene (PGR) variants and breast cancer risk in African American women. Breast Cancer Res Treat. 2013;139(3):833-43. https://doi.org/10.1007/s10549-013-2592-0.

48. Michels KB, Terry KL, Willett WC. Longitudinal study on the role of body size in premenopausal breast cancer. Arch Intern Med. 2006;166:2395-402.

49. Database of Single Nucleotide Polymorphisms (dbSNP). Bethesda (MD): National Center for Biotechnology Information, National Library of Medicine. dbSNP accession:\{rs11571153\}, (dbSNP Build ID: \{152\}). http://www.ncbi.nlm.nih.gov/SNP/

50. Iversen A, Frydenberg H, Furberg AS, Flote VG, Finstad SE, McTiernan A, Ursin G, Wilsgaard T, Ellison PT, Jasienska G, Thune I. Cyclic endogenous estrogen and progesterone vary by mammographic density phenotypes in premenopausal women. Eur J Cancer Prev. 2016;25:9-18.

51 Bertrand KA, Eliassen AH, Hankinson SE, Rosner BA, Tamimi RM. Circulating hormones and mammographic density in premenopausal women. Hormones Cancer. 2018;9:117-276.

52 Noh JJ, Maskarinec G, Pagano I, Cheung LWK, Stanczyk FZ. Mammographic densities and circulating hormones: a cross-sectional study in premenopausal women. Breast. 2006;15:20-8.

53 Boyd NF, Stone J, Martin LJ, Jong R, Fishell E, Yaffe M, Hammond G, Minkin S. The association of breast mitogens with mammographic densities. Br J Cancer. 2002;87:876-82.

54. Borugian MJ, Spinelli JJ, Gordon PB, Abanto Z, Brooks-Wilson A, Pollak MN, Warren LJ, Hislop TG, Gallagher RP. Fasting insulin and endogenous hormones in relation to premenopausal breast density (Canada). Cancer Causes Control. 2014;25:385-947.

55. Jung S, Stanczyk FZ, Egleston BL, Snetselaar LG, Stevens VJ, Shepherd JA, et al. Endogenous sex hormones and breast density in young women. Cancer Epidemiol Biomark Prev. 2014;24:369-78.

56. Hada M, Oh H, Fan S, Falk RT, Geller B, Vacek P, et al. Relationship of serum progesterone and progesterone metabolites with mammographic breast density and terminal ductal lobular unit involution among women undergoing diagnostic breast biopsy. J Clin Med. 2020;9:245.

Publisher's Note Springer Nature remains neutral with regard to jurisdictional claims in published maps and institutional affiliations. 